Findings 47 patients received standard treatment with tocilizumab. Total death was $44.7 \%$ in control group and $17 \%$ in tocilizumab group $(\mathrm{p}=0.008)$. $46.8 \%$ of patients in the tocilizumab group required ITU admission compared to $31.6 \%$ in the control group $(p=0.18) .27 .6 \%$ in tocilizumab needed intubation whereas $10.5 \%$ in control group $(p=0.06) .57 .5 \%$ in tocilizumab group were escalated to non invasive ventilation ( NIV) or high flow nasal oxygen (HFNO) whereas only $23.7 \%$ in control group required ventilatory support ( $\mathrm{p}$ value $=0.002)$. Further analysis of those in the ITU cohort revealed a mortality rate of $22.7 \%$ in the tocilizumab group and $58.3 \%$ in the control group . Length of hospital stay was twice in the tocilizumab group (12 days) vs control (6 days) $(\mathrm{p}<0.001)$.

Conclusion This study showed that tocilizumab may be associated with mortality benefit but no reduction in the rate of progression to intubation or need of NIV/HFNO. Further data with larger patient cohort is required to ascertain the benefits of tocilizumab in COVID 19 pneumonia.

\section{P187 CLINICAL OUTCOMES AND TREATMENT-RELATED ADVERSE EVENTS TO TOCILIZUMAB IN SARS-COV-2 ILLNESS}

S Ahmad, E Jenkinson, R Carney, T Nahu, J Quinn, A Dwarakanath. Mid Yorkshire Hospitals NHS Trust, Wakefield, UK

\subsection{6/thorax-2021-BTSabstracts. 296}

Background Severe SARS-CoV-2 is associated with release of Interleukin-6 and other pro-inflammatory cytokines that are markers of systemic inflammation and this response may cause or exacerbate lung injury leading to life-threatening disease. Tocilizumab, an IL-6 receptor antagonist licensed in certain Rheumatological disorders has shown to have beneficial effects on mortality and reduces the need for ventilator and organ support if used early. We evaluated the clinical outcomes and treatment \&ndash;related adverse events in patients who were treated with Tocilizumab.

Method A hospital treatment protocol with inclusion/exclusion criteria, dose regimen and clinical monitoring post dose and post discharge patient alert card (figure-1) was implemented. Two clinicians had to concur to the treatment and this was limited to patients needing either Acute Respiratory Care Unit (Level-1 HDU) or ICU. Electronic medical records of all patients who had Tocilizumab between January-May 2021 were reviewed. Baseline demographics, dose regimen, respiratory support at the time of treatment and adverse events were reviewed.

Results 108 patients (age; 56 $\pm 14, \mathrm{BMI} ; 32 \pm 3$, males-66\%) received Tocilizumab. The dosing regimen was weight based $(8 \mathrm{mg} / \mathrm{kg}$, maximum $800 \mathrm{mg})$ and was given within 24 hours in $79 \%$ patients and $21 \%$ within 48 hours of admission to either ARCU or ICU. Majority (95\%) received one dose and the second dose was only considered in the absence of clinical improvement. Respiratory support at the time of Tocilizumab treatment included CPAP -93\% (PEEP; 8-12), $1 \%$ nasal high flow therapy and $6 \%$ invasive ventilation including ECMO. Over a third of patients had no complications but $67 \%$ had deranged liver functions (elevated ALT) but settled with supportive measures, $1 \%$ had thrombocytopenia and $1 \%$ had reactivation of $\mathrm{TB}$ (TB Lymphadenitis). The mortality rate in patients who received Tocilizumab was

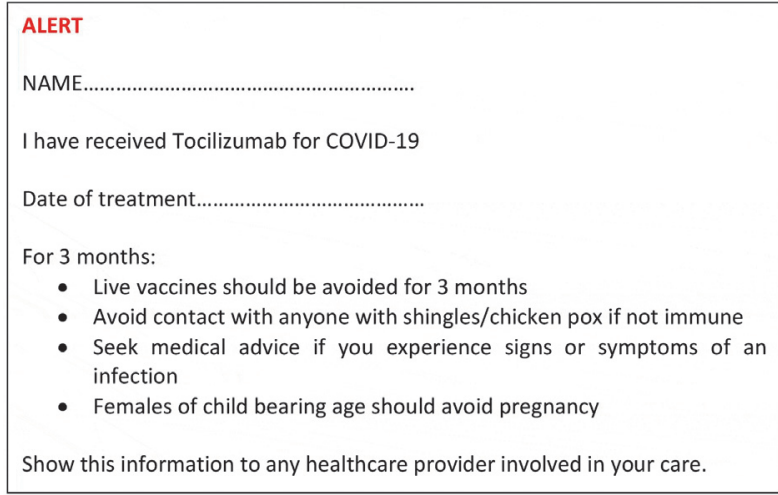

Abstract P187 Figure 1 Showing the post Tocilizumab alert card

24\% $(n=26)$. Post discharge alert card to the patients and specific discharge information to primary care were provided.

Conclusion Appropriate treatment protocol and regular monitoring are needed for patients who receive Tocilizumab in severe SARS-CoV-2 illness. Clinicians should bear in mind the high incidence of treatment-related adverse events and the lack of data about long term effects. Treatment alert cards and specific discharge advice may be beneficial.

\section{P188 TICK TOCK...WHERE AND WHEN CAN WE GIVE TOC? REVIEW OF COVID-19 PATIENTS RECEIVING TOCILIZUMAB IN A NON CRITICAL CARE SETTING}

K Aiken, M Wilson, E Keelan. Mater Hospital, Belfast, UK

\subsection{6/thorax-2021-BTSabstracts.297}

Introduction The REMAP-CAP trial demonstrated the positive effects of interleukin-6 receptor antagonists (tocilizumab and sarilumab), on mortality in COVID-19 patients managed in a critical care setting. ${ }^{1}$ Prior to this, adjuvant drug therapies such as remdesivir and dexamethasone have shown limited benefits regarding COVID-19 related mortality in patients requiring non-invasive respiratory, managed in non-critical care settings.

During the pandemic the Mater Hospital, Belfast was designed as the local COVID-19 centre. Prior to January 2021 standard ward level care included IV antibiotics, IV remdesivir, oral dexamethasone and non-invasive ventilation. Continuous positive airway pressure was used first line (commenced when $\mathrm{FiO} 2$ requirements exceeded $4 \mathrm{~L} / \mathrm{min}$ via nasal cannula). After the release of the Department of Health's position statement regarding tocilizumab for COVID-19 patients on respiratory support, a decision was taken to use tocilizumab off license in a non-critical care setting.

Our hypothesis was that COVID-19 positive patients on non-invasive ventilation who received tocilizumab in addition to standard care would have reduced hospital mortality compared with standard care alone. The REMAP-CAP trial administered tocilizumab to COVID-19 patients in a critical care setting, however we postulated that those 'less unwell' patients requiring ward level respiratory support but not 'critical care' could still benefit.

Methods Patients commenced on tocilizumab in a non-critical care setting were identified and followed up prospectively. A 
control group receiving ward level standard care was established retrospectively.

Results Forty patients were recruited into both the control and treatment groups. Results were analysed using Chi-squared statistics on Microsoft Excel. The primary outcome, namely; hospital mortality, demonstrated a significant difference between the groups $(p=0.048)$ with no discernible difference in side effect profile.

Conclusion This data supports the use of tocilizumab in patients with COVID-19 disease, noting its positive effect on hospital mortality for COVID-19 patients on non-invasive respiratory support but not requiring critical care. Moreover, the limited side effect profile witnessed suggests tocilizumab can be safely administered in a non-critical care setting.

\section{REFERENCE}

1. Gordon AC, Mouncey PR, Al-Beidh F, Rowan KM, Nichol AD, Arabi YM, et al. Interleukin-6 receptor antagonists in critically ill patients with COVID-19-preliminary report. medRxiv. 2021.

\section{P189 USE OF ANGIOTENSIN-CONVERTING-ENZYME INHIBITORS AND ANGIOTENSIN RECEPTOR BLOCKERS IN COVID-19 INFECTION DOES NOT ADVERSELY AFFECT CLINICAL OUTCOMES INCLUDING NEED FOR NON- INVASIVE AND INVASIVE VENTILATION}

A Amrapala, M Win, A Ainley. Barking, Havering and Redbridge University Hospitals NHS Trust, London, UK

\subsection{6/thorax-2021-BTSabstracts. 298}

Introduction It has been hypothesized that use of AngiotensinConverting-Enzyme Inhibitors (ACE-I) and Angiotensin Receptor Blockers (ARB) are associated with worse outcomes in COVID-19 through upregulation of ACE2 receptors. ${ }^{1}$ Recent studies have shown no association between ACE-I/ARB use and increased mortality but there is limited information on other markers of disease severity such as need for Non-invasive and invasive ventilation. We assessed the effect of ACE-I/ ARB on the outcomes of COVID-19 patients.

Methods A retrospective observational cohort study of patients with confirmed COVID-19 admitted to the respiratory units during a 1-year period. Patient demographics, clinical and medication history and clinical outcomes were extracted from written and electronic records. Primary outcomes - length of stay (LOS), NIV requirement, intensive care (ITU) admission, intubation and death - were compared between those who received ACE-I/ARB concurrently with their COVID-19 treatment and those who did not. Statistical analysis was performed using chi-squared test and odds ratio (OR).

Results Of 521 patients with confirmed COVID-19 (median age 59 years, $62.6 \%$ male), $183(35.1 \%)$ required NIV, 108 $(20.7 \%)$ were admitted to ITU, $60(11.5 \%)$ were intubated and $41(7.9 \%)$ died. In total, 151 (29\%) were on ACE-I/ARB treatment, most commonly for hypertension. There was no difference in median LOS between those on ACE-I/ARB treatment and those not (11 and 10 days respectively, $p=0.20)$. There was no difference between NIV requirement (OR 1.13, 95\% CI 0.71-1.56), admission to intensive care (OR 0.64, 95\% CI 0.50-1.36), intubation (OR 0.65 , 95\% CI $0.43-1.58$ ) and death (OR 1.15, 95\% CI $0.53-2.11$ ) between the two groups $(p>0.05)$.
Conclusion There was no difference in clinical outcomes between COVID-19 patients on ACE-I/ARB and those who were not, in particular with regards to need for non-invasive and invasive ventilation. Our findings support current recommendations for continued use of ACE-I/ARB in COVID-19 infection.

\section{REFERENCE}

1. Fang $L$, et al. Are patients with hypertension and diabetes mellitus at increased risk for COVID-19 infection? The Lancet. Respiratory medicine 2020; 8(4):e21. doi:10.1016/S2213-2600(20)30116-8

\section{P190 THE IMPACT OF DRUG THERAPIES ON COVID-19 MORTALITY IN A UK TERTIARY CENTRE}

${ }^{1} \mathrm{~T}$ Kurmoo, ${ }^{1} \mathrm{M}$ Mayisha Ahmad, ${ }^{2} \mathrm{R}$ Wang, ${ }^{2} \mathrm{~N}$ Chaudhuri. ${ }^{1}$ University of Manchester, Manchester, UK; ${ }^{2}$ Manchester Foundation Trust, Wythenshawe, Manchester, UK

\subsection{6/thorax-2021-BTSabstracts.299}

Introduction and Objectives The availability of treatment options for Covid-19 is rapidly expanding. Whilst the efficacy data is well-established from clinical trials, real-life efficacy of drug therapies remains lacking. We aimed to compare clinical outcomes between first and second wave of Covid-19 and determine real-world effectiveness of dexamethasone on 30day mortality.

Methods This is a retrospective observational study. Clinical data and information regarding 30-day mortality, length of stay (LOS) and Intensive Care Unit (ICU) admission of hospitalised Covid-19 patients during early first wave (10.03.2020 to 13.04 .2020$)$ and second wave $(01.12 .2020$ to 09.02 .2021$)$ were collected. Treatment was limited to second wave and included either dexamethasone only or both remdesivir and dexamethasone. The effectiveness of dexamethasone only on 30-day mortality was measured.

Results Of 373 patients (64.3\% male) during the first wave, $24.9 \%$ died within 30 days. The 30 -day mortality rate was lower during the second wave $(61 / 324,18.8 \%$, p-value $=$ 0.064). Patients were younger (mean [SD], 60.0 [16.5] years) and had higher body mass index (mean [SD], 30.3 [11.0] kg/ $\mathrm{m}^{2}$ ) during the second wave than the first wave (68.7 [14.8] years and $\left.28.2[7.70] \mathrm{kg} / \mathrm{m}^{2}\right)$. In the first wave, no patients received specific drug therapy for Covid-19. However, 86.5\% of patients received dexamethasone only during the second wave. The LOS for the first wave was longer (median (IQR): 5 (2-11) days) compared to the second wave (4 (2-9) days, $\mathrm{p}=0.013)$. ICU admission during the second wave $(11.2 \%)$ was also lower than the first wave $(23.4 \%, \mathrm{p}<0.001)$. In second wave, $14.3 \%$ of patients who were given dexamethasone died within 30 days compared to $25 \%$ who had no treatment ( $\mathrm{p}$-value $=0.088$ ).

Conclusions In the real-world setting, there was an improvement in mortality, shortened hospital LOS and lower ICU admission rate between early first and second waves of the pandemic. The major difference in treatment strategy between the two waves was the approval of drug therapies in hospitalised patients. Dexamethasone reduced the 30-day mortality, although it did not reach statistical significance, likely due to the retrospective nature and small sample size of this study. Our findings corroborate clinical trial data on the benefit of dexamethasone therapy. 